\title{
Burning Mouth Syndrome: A Brief Review
}

\author{
Sadeq A. Al-Maweri ${ }^{1}$, Abdulrahman Abdullah Alhagbani ${ }^{2}$, Ahmed Saleh \\ Alanazi $^{3}$, Abdullah Farhan ${ }^{4}$, Eman Al-Mawri ${ }^{5}$ \\ ${ }^{1}$ (Department of oral Medicine and Diagnostic Sciences, Alfarabi Colleges, Saudi Arabia) \\ ${ }^{2}$ (Dental center, Alnakheel primary care center, Riyadh, Saudi Arabia) \\ ${ }^{3}$ (Undergraduate student, Alfarabi Colleges, Saudi Arabia) \\ ${ }^{4}$ (Department of oral and Maxillofacial Surgery, Sana'a University, Yemen) \\ ${ }^{5}$ (Department of oral Medicine, Sana'a University, Yemen)
}

\begin{abstract}
Burning mouth syndrome (BMS) is a common chronic pain condition of unknown etiology and indefinite cure. It mainly affects postmenopausal women at their 5th to 7th decade. It has a grave impact on quality of life, interfering with eating, drinking and other daily functions. Although the etiology remains obscure, recent evidence suggests BMS to be a neuropathic in nature. Diagnosis of BMS is quiet challenging for dental practitioners and is basically based on careful analysis of symptoms reported by the patient. There is no specific treatment for BMS as yet and the treatment is aimed solely to alleviate symptoms without definite cure. There are a number of treatments available for BMS including antidepressants, anticonvulsants, benzodiazepines, analgesics, dietary supplements, and topical anesthetic agents. This article aimed to briefly review the literature regarding BMS with a particular emphasis on different approaches of the management.
\end{abstract}

Keywords: Burning mouth syndrome, diagnosis, management

\section{Introduction}

Burning mouth syndrome (BMS) is a chronic pain condition, predominantly affecting post-menopausal women $(1,2)$. According to the International Headache Society, burning mouth syndrome (BMS) is defined as an intraoral burning sensation for which no medical or dental cause can be identified (3). Other terms used in the literature to describe BMS are glossodynia, glossopyrosis, stomatopyrosis, stomatodynia, and dysesthesia(4). The patient usually complains of burning sensation, unremitting mucosal pain, xerostomia and dysguesia (5) . BMS often affects the tongue (especially the anterior two thirds), lower lip and hard palate, although any site of oral mucosa can be affected $(5,6)$. BMS is a relatively common condition with an estimated prevalence rate of $2.6 \%$ in the general population, and up to $12-18 \%$ among postmenopausal women $(5,6)$, with a female to male ratio of 7-1 (6). The aim of this article was to briefly review the current literature regarding BMS with a particular emphasis on the current treatment strategies.

\section{Etiology and pathogenesis}

Although the exact etiopathogenesis of BMS is uncertain, it seems to be multifactorial condition that involves interactions between local, systemic, and psychological factors $(1,5,6)$. However, recent evidence suggests that BMS is neuropathogenic in nature, and neurophysiological studies indicates the possibility of a dysfunction at the peripheral and central reflex arc path and the processing of cortical excitation $(7,8)$. Lauria and colleagues examined oral muocal biopsies obtained from the lateral surfaces of the anterior tongue of BMS patients, and found that patients with BMS demonstrated decreased density of epithelial nerve fibers as well as axonal derangement, indicating a potential role for peripheral small-fiber sensory neuropathy (9).

\section{Clinical Features And Diagnostic Criteria}

The diagnosis of BMS needs a careful analysis of the symptoms experienced by the patient. The prime feature of BMS is a deep continuous oral mucosal burning sensation or pain, unremitting for at least 4-6 months, in the absence of any visible mucosal alteration or abnormal laboratory findings $(1,5,10)$. The oral burning or pain rarely interferes with sleep and never worsens, but may be relieved, by eating and drinking. Other accompanied symptoms that can help identify BMS include: xerostomia, taste alteration, mood changes, and specific disruptions in patient personality traits $(1,5,6)$. BMS patients can not have any pathological abnormalities such as ulcer, white lesion, erythema, atrophy, erosion, and stomatitis Diagnosis of BMS is still a quiet challenging task for clinicians, and the clinical diagnosis basically relies on a thorough review of patient's medical and dental histories, and a careful analysis of physical and laboratory findings. 


\section{Management of BMS}

Given the obscure etiology of BMS, no specific treatment exists as yet and the treatment is solely symptomatic. The most common medications used for treatment BMS are tricyclic antidepressants, benzodiazepines such as clonazepam, anticonvulsants such as Gabapentin, analgesics, and topical capsaicin (Table 1) $(10,11)$. Other proposed treatment modalities include vitamins, dietary supplements, hormonal replacements, topical estrogen (in case of post-menopausal women), and psychotherapy (11). However, despite the large variety of medication and other approaches proposed for BMS management, the treatment has been unsatisfactory and as yet there is no a definite cure. Moreover, due to chronicity of the condition, which may last for several years and the fact that more than one medication (combination therapy) is often used for treatment of BMS, the treatment has several devastating adversary effects. Recently, laser therapy has been suggested and used for treatment of pain in BMS patients. Low-level laser therapy (LLLT), also known as photobiomodulation is a non-drug, non-invasive clinical application that has been used for treatment of inflammatory disorders (10). It has potential analgesics, anti-inflammatory, immunomodulating and biostimulating effects (12). It has demonstrated several therapeutic effects that can be used for treatment of burning sensation induced by BMS. Currently, there is a growing increase in the use of LLLT in different areas of dentistry especially for treatment of chronic inflammatory and painful conditions such as BMS, oral mucositis , myofacial pain and chronic neck pain, and temporomandibular joint disorders $(2,13,14)$. A recent systematic review by Al-Maweri and colleagues (2) has evaluated the efficacy of laser therapy in treatment of BMS. The study concluded that LLLT is effective in treatment of BMS without any reported adverse effects. However the authors suggested that the lack of consensus on the ideal laser parameters creates a barrier in establishment of an ideal laser-based treatment protocol for BMS; and consequently more well designed studies, taking in consideration the ideal laser parameters based on the analysis of the best available evidence are warranted.

Figures and Tables

Table 1: Different therapy modalities used for treatment of BMS

\begin{tabular}{|c|}
\hline Type of therapy \\
\hline Systemic Pharmacological therapy \\
$>$ Tricyclic antidepressants: Amitriptyline $10 \mathrm{mg}$ \\
$>$ Benzodiazepine: Clonazepam $0.252 \mathrm{mg}$ \\
$>$ Anticonvulsants: Gabapentin $100 \mathrm{mg}$ \\
$>$ mg \\
$>$ Hormonal replacements \\
$>$ Dietary supplements: Folate \\
Topical Pharmacological therapy \\
$>$ Lidocain \\
$>$ Clonazepam \\
$>$ Capcetin \\
$>$ Benzydamine hydrochlorate \\
\hline Non-pharmacological treatment \\
$>$ Laser therapy \\
$>$ Laser acupuncture \\
$>$ Psychotherapy \\
\hline
\end{tabular}

\section{Conclusion}

Burning mouth syndrome is a complex pain condition that has a negative impact on oral health-related quality of life of patients. Treatment of BMS remains unsatisfactory and there is no definitive cure. Therefore, a multidisciplinary approach is usually required to alleviate the symptoms. A variety of topical and systemic medications have been widely used for BMS treatment including antidepressants, antipsychotics, and benzodiazepines. Non-pharmacological approaches such as psychotherapy, and cognitive therapy and laser irradiation have also been suggested and used for treatment of this condition. Our opinion is that more research is needed to better understand the etiopathogenesis of BMS, and more well-designed clinical trials are required to assess the efficacy of newly suggested therapies. 


\section{References}

[1]. Beneng K, Renton T. Pain Part 8: Burning Mouth Syndrome. Dental update. 2016;43(3):254-6, 9-62, 65-6.

[2]. Al-Maweri SA, Javed F, Kalakonda B, AlAizari NA, Al-Soneidar W, Al-Akwa A. Efficacy of Low Level Laser Therapy in the Treatment of Burning Mouth Syndrome: A Systematic Review. Photodiagnosis and photodynamic therapy. 2017;17:188-93

[3]. Headache Classification Subcommittee of the International Headache Society. The International Classification of Headache Disorders: 2nd edition. Cephalalgia. 2004;24 Suppl 1:9-160.

[4]. Yang HW, Huang YF. Treatment of burning mouth syndrome with a low-level energy diode laser. Photomedicine and laser surgery. 2011;29(2):123-5.

[5]. Sun A, Wu KM, Wang YP, Lin HP, Chen HM, Chiang CP. Burning mouth syndrome: a review and update. Journal of oral pathology \& medicine. 2013;42(9):649-55.

[6]. Scala A, Checchi L, Montevecchi M, Marini I, Giamberardino MA. Update on burning mouth syndrome: overview and patient management. Critical reviews in oral biology and medicine. 2003;14(4):275-91.

[7]. Spanemberg JC, Lopez Lopez J, de Figueiredo MA, Cherubini K, Salum FG. Efficacy of low-level laser therapy for the treatment of burning mouth syndrome: a randomized, controlled trial. Journal of biomedical optics. 2015;20(9):098001.

[8]. Yilmaz Z, Renton T, Yiangou Y, Zakrzewska J, Chessell IP, Bountra C, et al. Burning mouth syndrome as a trigeminal small fibre neuropathy: Increased heat and capsaicin receptor TRPV1 in nerve fibres correlates with pain score. Journal of clinical neuroscience. 2007;14(9):864-71.

[9]. Lauria G, Majorana A, Borgna M, Lombardi R, Penza P, Padovani A, et al. Trigeminal small-fiber sensory neuropathy causes burning mouth syndrome. Pain. 2005;115(3):332-7.

[10]. Lopez-Jornet P, Camacho-Alonso F, Andujar-Mateos P, Sanchez-Siles M, Gomez-Garcia F. Burning mouth syndrome: an update. Medicina oral, patologia oral y cirugia bucal. 2010;15(4):e562-8.

[11]. Kisely S, Forbes M, Sawyer E, Black E, Lalloo R. A systematic review of randomized trials for the treatment of burning mouth syndrome. Journal of psychosomatic research. 2016;86:39-46.

[12]. Pandeshwar P, Roa MD, Das R, Shastry SP, Kaul R, Srinivasreddy MB. Photobiomodulation in oral medicine: a review. Journal of investigative and clinical dentistry. 2016;7(2):114-26.

[13]. Bjordal JM, Bensadoun RJ, Tuner J, Frigo L, Gjerde K, Lopes-Martins RA. A systematic review with meta-analysis of the effect of low-level laser therapy (LLLT) in cancer therapy-induced oral mucositis. Supportive care in cancer. 2011;19(8):1069-77.

[14]. Pinheiro AL, Cavalcanti ET, Pinheiro TI, Alves MJ, Miranda ER, De Quevedo AS, et al. Low-level laser therapy is an important tool to treat disorders of the maxillofacial region. Journal of clinical laser medicine \& surgery. 1998;16(4):223-6. 